\title{
Noise and Vibration Analysis of a Heat Exchanger: a Case Study
}

\author{
Thiago A. Fiorentin and Alexandre Mikowski \\ Department of Mobility Engineering, Federal University of Santa Catarina, Joinville, Brazil 88040-900
}

\author{
Olavo M. Silva and Arcanjo Lenzi \\ Federal University of Santa Catarina, Florianopolis, Brazil 88040-900
}

(Received 15 November 2016; accepted: 26 April 2017)

Flow-induced vibration of heat-exchanger tube bundles often causes serious damage, resulting in reduced efficiency and high maintenance costs. The excitation mechanism of flow-induced vibration is classified as vortex shedding, acoustical resonance, turbulent buffeting, or fluid-elastic instability. This paper aims to identify the mechanism that causes flow-induced vibration in a specific heat-exchanger tube bundle with cross-flow and proposes a solution to this problem. This case is investigated through acceleration and sound pressure level measurements. Moreover, finite- element models are developed to view the acoustic models of the cavity and vibration modes of the tubes and plates. The layout pattern of the tube array, the spacing ratio, the Strouhal number, and the flow characteristics are used to determine the excitation mechanism.

\section{INTRODUCTION}

In cogeneration mills, the excess of energy generated can be transferred to an electrical system. ${ }^{1}$ In an attempt to improve the efficiency of these systems, some manufacturers have developed new heat exchangers in which the speed of the fluid flowing around the tubes has been increased; they have also changed their tube arrangements. The production cost of major heat-exchanger equipment is high. Also, since maintenance is necessary, the cost of the entire operation is quite high. According to the literature, many incidents of failure due to apparent flow-induced vibration in heat exchangers have been reported. $^{2}$

Flow-induced vibration and acoustical resonance have caused serious damage to the system integrity of heat exchangers. ${ }^{3}$ The four principal sources of vibration in cross-flow tube banks are vortex shedding, acoustical resonance, turbulent buffeting and fluid-elastic instability. ${ }^{4,5}$ All these mechanisms arise because of the various forces that act on a tube due to the shell-side cross-flow.

During the last decades, researchers were successful (in varying degrees) in better understanding the main sources of noise and vibration in different kind of heat exchangers. 6 ,9 Distinct solutions have been proposed to control the noise and vibration problem in heat exchangers. In order to suppress acoustical resonances, a rigid baffle is normally placed inside a container and is parallel to the flow stream. It modifies the acoustical field and inhibits the instability. ${ }^{10}$ To avoid the vibration of the equipment, it is sometimes necessary to reduce the shell side flow rate, remove the tubes in the window area to form a bypass, and redesign and reinstall a new bundle. ${ }^{11}$

However, it is difficult to find case histories presenting experimental analyses, finite element models, and a solution to the problem. Normally, the investigations are focused on excitation mechanisms, instabilities criteria, and methods to predict the problem.

\section{EXCITATION MECHANISMS}

Recently, a significant amount of research has been conducted to predict, understand, and resolve the mechanisms that

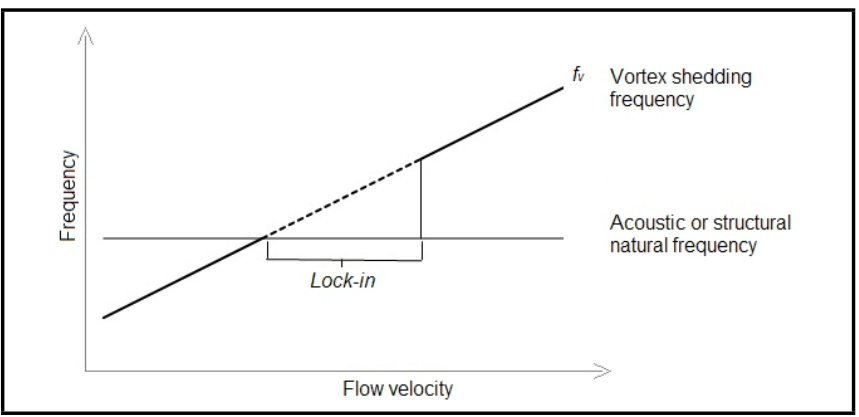

Figure 1. Lock-in condition.

cause flow-induced vibration in heat exchangers. The following four main mechanisms of flow-induced vibration in heat exchangers have been investigated.

\subsection{Vortex Shedding}

Flow across a tube produces vortices in the wake generated from the opposite sides of the tube. The oscillation frequency of a wake is proportional to the flow velocity and results in an oscillatory force on the tube. This phenomenon may excite vibration in a liquid flow or acoustical resonance in a gas flow.

When the frequency of the vortex shedding coincides with the natural frequency of the tube vibration, higher levels of vibrations and rapid tube damage may occur, especially for liquid flows. The velocity range over which the tubes exhibit high-amplitude vibrations is referred to as the "lock-in" range, as shown in Fig. 1. The lock-in conditions can be estimated from the following equation:

$$
0.8 f_{n}<f_{v}<1.2 f_{n}
$$

with

$$
f_{v}=S t_{v} \frac{U}{D}
$$

where $f_{n}$ is the tube's natural frequency, $f_{v}$ is the frequency of vortex shedding, $S t_{v}$ is the Strouhal number, $U$ is the gap velocity, and $D$ is the tube diameter

Recent studies have shown that this excitation results from vortex shedding around the tubes. It occurs in the beginning of 


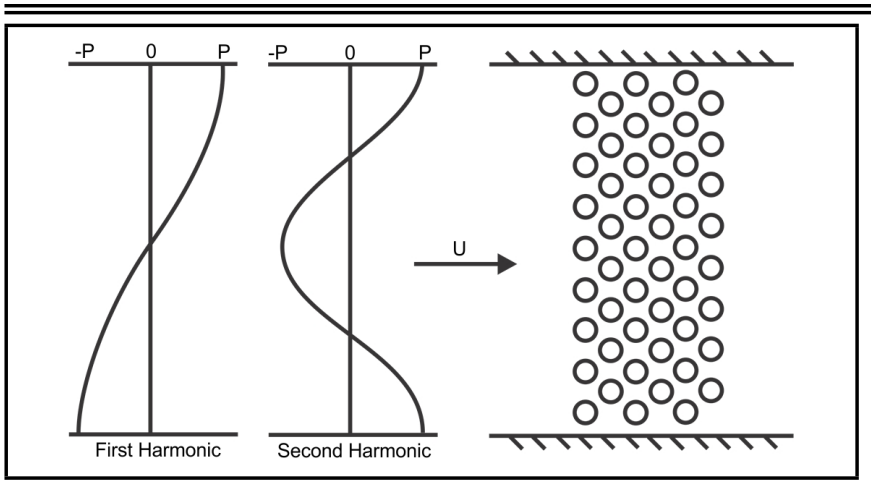

Figure 2. Acoustial modes inside the heat exchanger.

the section tubes, mainly in heat exchangers with little space between tubes. ${ }^{12,13}$

\subsection{Acoustical Resonance}

Acoustical resonance excited by a flow can occur in different types of heat exchangers. Normally, they have tubes with fluid flow inside (tube-side fluid) and a shell that wraps around the tubes (shell-side fluid). The gas in the cavity of the heat exchanger generates natural acoustic frequencies that depend on the speed of sound and the shell geometry. These acoustic modes can be excited by either vortex shedding or turbulent buffeting.

This resonance, if and when excited, is established within the tube bundle in a direction perpendicular to the flow and the tube axis, as shown in Fig.2. For a cylindrical heat exchanger, the natural acoustic frequencies can be predicted by the following equation:

$$
f_{a}=\frac{n U_{s}}{2 d}
$$

where $f_{a}$ is the acoustic frequency, $n$ is the mode number, $d$ is the shell diameter, and $U_{s}$ is the velocity of sound in shell-side fluid. ${ }^{14}$

Acoustical resonance requires two conditions: (i) coincidence of vortex shedding and acoustic frequency and (ii) sufficiently high acoustic energy or sufficiently low acoustic damping to allow sustained acoustic standing-wave resonance. ${ }^{15}$ An acoustical resonance can be broken by inserting acoustic baffles into the shell. These baffles can change the natural acoustic frequency and the mode shape.

\subsection{Turbulent Buffeting}

High flow rates produce turbulent flow within a fluid, which can be a cause of structural excitation. In any flow, there exists a range of oscillatory flow components spread over a broad range of frequencies. Tubes respond randomly to flow turbulence. Turbulent buffeting is a low-amplitude vibration response of the tube bundle below a certain critical velocity. ${ }^{16}$

Although the vibration amplitudes are small, the phenomenon persists over the entire life of the heat exchanger, because some wear is inevitable. Even when other vibration mechanisms are successfully mitigated, this turbulent buffeting may eventually cause tube damage. For the heat exchanger to remain in operation for many years, the potential for wear due to turbulent buffeting needs to be considered in the design stage of the heat exchanger.

Flow turbulence is a significant excitation mechanism in a heat exchanger with cross-flow. The dominant frequency of excitation can be determined by Owen's equation: ${ }^{17}$

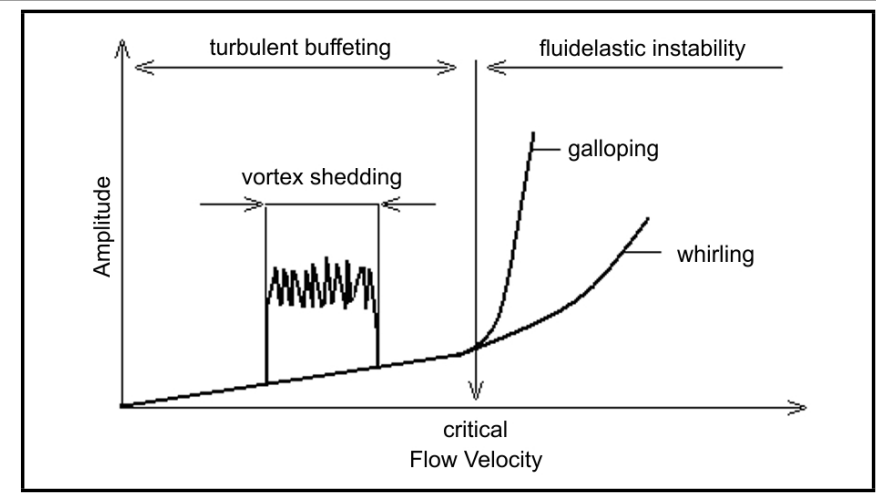

Figure 3. Fluid-elastic instability, turbulent buffeting, and vortex shedding.

$$
S t_{t b}=\frac{f_{t b} D}{U} X_{l} X_{t}=\left[3.05\left(1-\frac{D}{L_{t}}\right)^{2}+0.28\right]
$$

where $S t_{t b}$ is the Strouhal number considering turbulent buffeting, $f_{t b}$ is the turbulent buffeting frequency, $U$ is the crossflow velocity, $D$ is the outer diameter of the tube, $X_{l}$ is the longitudinal pitch ratio $\left(\frac{L_{l}}{D}\right), X_{t}$ is the transverse pitch ratio $\left(\frac{L_{t}}{D}\right), L_{l}$ is the longitudinal pitch and $L_{t}$ is the transverse pitch.

\subsection{Fluid-Elastic Instability}

Fluid-elastic instability can be defined as a mechanism where fluid forces cause tube movement in the heat exchanger. At a critical flow velocity, tube banks subjected to increasing flow velocity begin to vibrate with a large amplitude, as shown in Fig. 3. The resultant tube failure can occur in a relatively short period due to fluid-elastic instability, which can be avoided by controlling the cross-flow velocity.

The flow field around a tube bundle causes displacement of a tube. This displacement modifies the flow field, changing the fluid forces acting on the tube. Damping and stiffness forces of the tube act to restore the tube to its initial position. The competition between the energy input from the fluid flow across the tube and the energy dissipated by damping and stiffening will determine the amplitude of the vibration. If the energy dissipated by damping and stiffening is less than the energy input from the fluid flow, then fluid-elastic vibrations will establish themselves.

Connor's equation is used as a parameter to verify the possibility of developing fluid-elastic instability in a tube array. The equation below calculates the critical flow velocity based on tube parameters:

$$
\frac{U_{c r}}{f_{n} D}=K\left(\frac{m \delta}{\rho D^{2}}\right)^{a}
$$

where $U_{c r}$ is the critical pitch flow velocity, $D$ is the diameter of the tube, $f_{n}$ is the natural structural frequency, $m$ is the tube mass per unit length, $\rho$ is the density, $\delta$ is the logarithmic decrement, and $K$ and $a$ are Connor's constants. ${ }^{18}$

\section{EXPERIMENTAL AND NUMERICAL ANALYSIS}

The heat exchanger under analysis is a cross-flow type with a geometric tube distribution, as shown in Fig. 4. The tubes have an external diameter of $63 \mathrm{~mm}$, length of $3.80 \mathrm{~m}$ and a 4$\mathrm{mm}$-thick wall. They are distributed over 26 rows by 54 rows. 


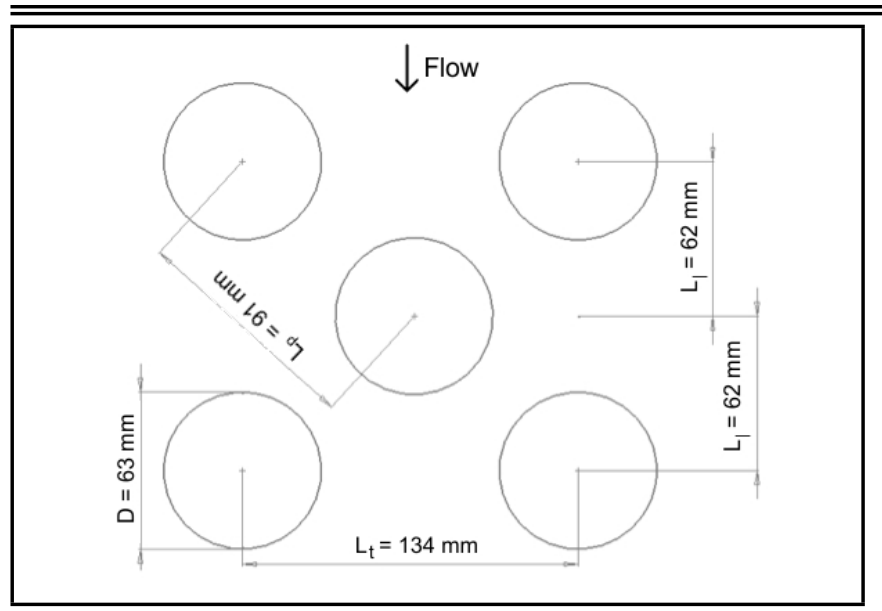

Figure 4. Cross-section of tube geometric distribution.

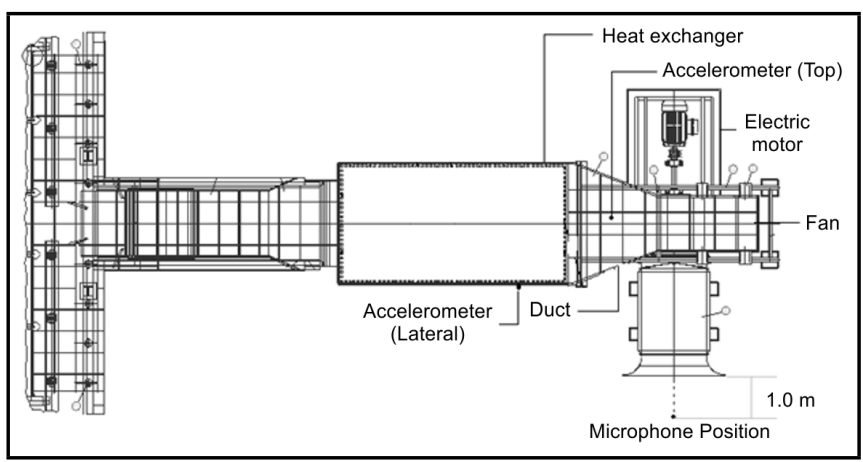

Figure 5. Accelerometer and microphone positions in the heat exchanger.

The heat exchanger is $3.80 \mathrm{~m}$ in height, $3.50 \mathrm{~m}$ in width, and $6.70 \mathrm{~m}$ in length.

A $350 \mathrm{hp}$ fan blower insufflates an airflow through the heat exchanger. To identify the cause of excessive noise and vibration, the rotational speed of the fan is varied from 450 to 750 $\mathrm{rpm}$, which corresponds to an upstream air velocity of 2.90 to $5.00 \mathrm{~m} / \mathrm{s}$, respectively. The inlet air temperature is $25^{\circ} \mathrm{C}$ and the air temperature at the end of the heat exchanger is $240^{\circ} \mathrm{C}$.

According to the configuration of the heat exchanger analysed in this paper, the space ratio $X_{p}\left(\frac{L_{p}}{D}\right)=1.44$, the Strouhal number from the literature is $S t=1.0^{19}$ and the Strouhal number considering turbulent buffeting $S t_{t b}=1.14$, as calculated by Eq. (4).

\subsection{Experimental Analysis}

To help with the problem identification, noise and vibration measurements are performed at various speeds of the primary fan blower, resulting in a range of mean flow velocities in the heat exchanger. This procedure is used to analyse situations when the excitation frequency coincides with the acoustic or structural modes of vibration.

The vibration is measured on the side wall of the heat exchanger inlet duct. The sound pressure level (SPL) is measured in front of the blower inlet nozzle, distant 1.0 meter of fan attenuator. The vibration is measured on the side wall of the exchanger inlet duct. The sound and vibration measurements are made simultaneously. These quantities are measured for different fan rotation values. The microphone and accelerometer position, as well as the main components of the heat exchanger can be identified at Fig. 5.

Analysing the results shown in Figs. 6 and 7 enables the identification of the highest amplitude of the frequency gener-

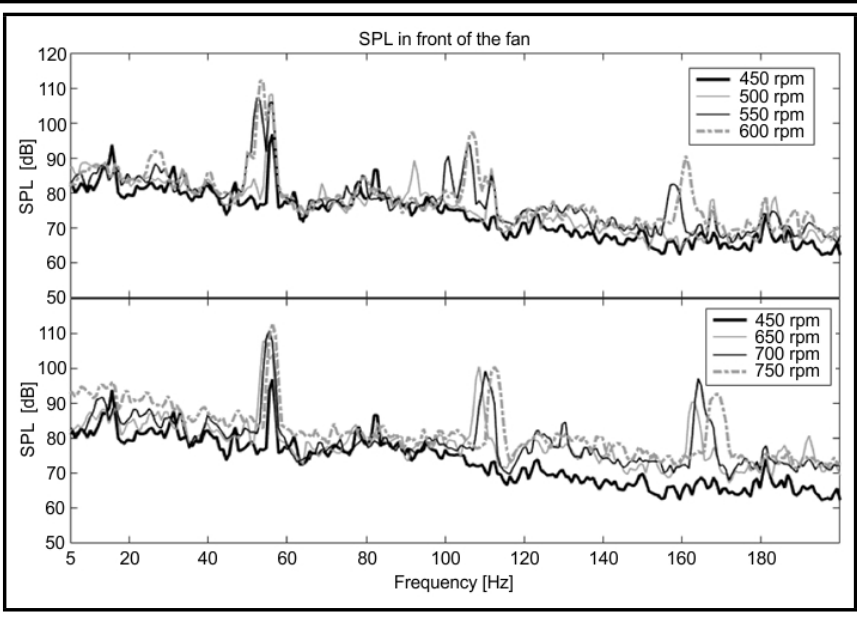

Figure 6. Sound pressure level measured in front of the fan inlet.

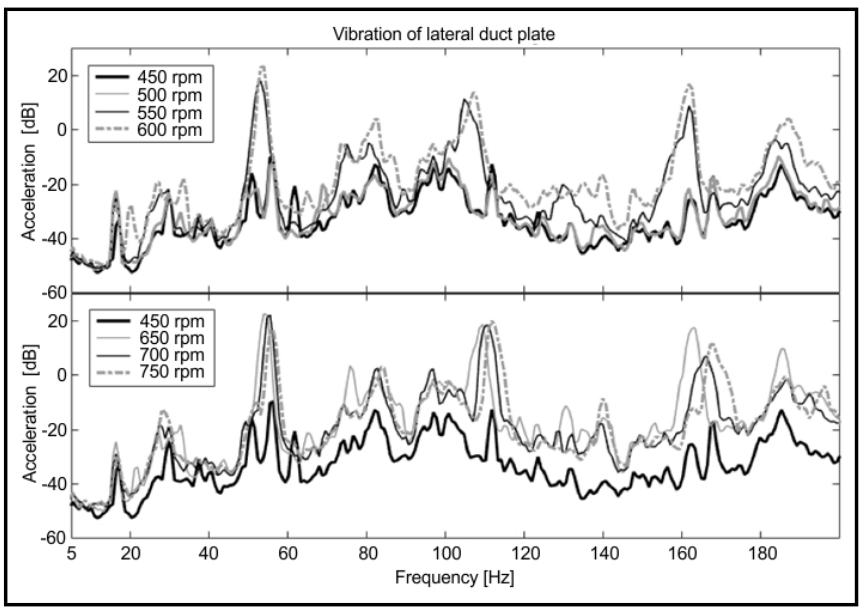

Figure 7. Acceleration measured on the lateral plate.

ated as approximately $53 \mathrm{~Hz}$ along with its resultant harmonics.

\subsection{Numerical Analysis}

The correct identification of the mechanism that generates noise and vibration requires a precise knowledge of the natural acoustic frequencies of the tubes and their respective vibration modes. Numerical models are developed to analyse the vibrations of the tubes, the side plate, and the acoustical field in the heat exchanger.

\subsubsection{Tube Vibrations}

A bundle of tubes is modelled by a finite-element method using a shell element. These tubes are coupled to 20 -mm-thick plates at the ends. When the tubes are welded to the plate, it is considered to be a rigid connection. In this model, a larger number of tubes produce a slow computational solution. It is assumed that the model can reproduce the true behaviour of the tubes in the heat exchanger. Due to the fixation of the tubes in a rigid structure, the behaviour of this system is associated with the modes located on each tube. The steel damping coefficient is defined as $0.05 \%$.

The objective of the model is to obtain the frequency ranges of the natural vibration modes. Figure 8 demonstrates that it is possible to identify a typical frequency response for each tube excited by a point force. The first three modes are plotted in Figs. 8 and 9.

There is no peak at $53 \mathrm{~Hz}$; however, the second mode of vibration occurs between 62 and $68 \mathrm{~Hz}$. Experimentally, the 


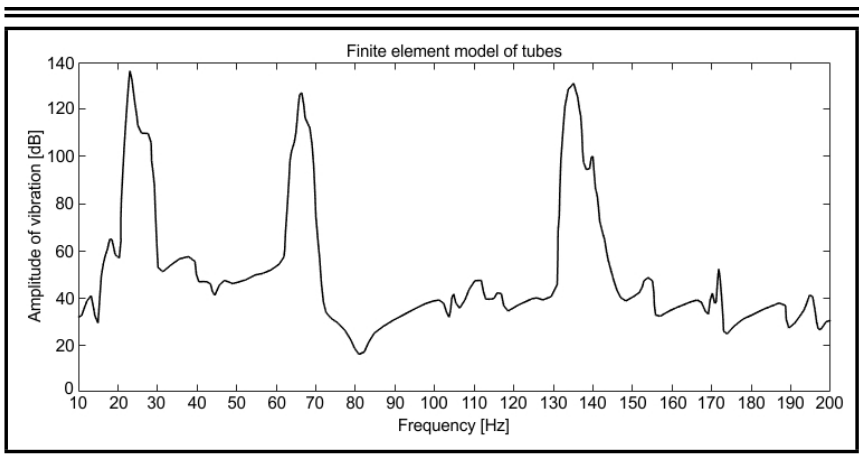

Figure 8. Frequency response function of tubes.

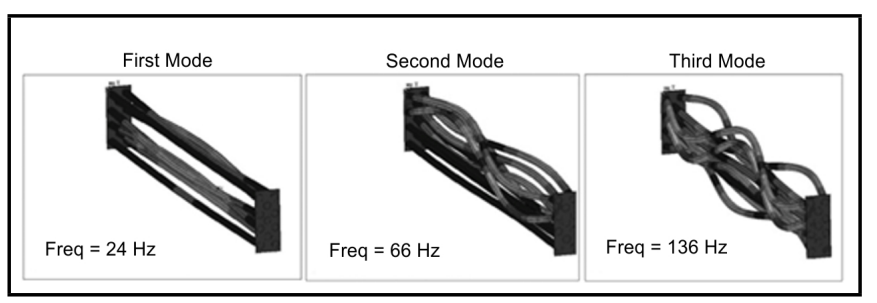

Figure 9. Modes of vibration.

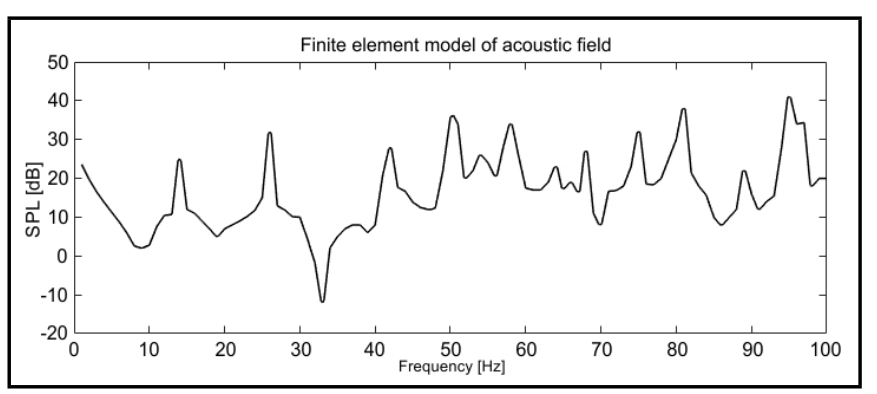

Figure 10. Frequency response function of the acoustical field.

modes occur at $21 \mathrm{~Hz}, 58 \mathrm{~Hz}$, and $118 \mathrm{~Hz}$. The excitation is applied at one side of the tube e the response measured at the other side of the tube.

\subsubsection{Acoustic Modes}

This simulation considers air with a variable temperature distribution over the length of the exchanger, from $30^{\circ} \mathrm{C}$ at the blower exit up to $225^{\circ} \mathrm{C}$ at the end of the exchanger. The finite-element method can be used to consider the temperature variation in the acoustical field of the exchanger. The speed of sound in the longitudinal and transversal directions is considered to be $95 \%$ of that in air due to the porosity caused by the tubes. In the model, there is no coupling between the acoustic and structural components. A typical frequency response function can be observed in Fig. 10.

The boundary condition defined at fan inlet is a panel velocity. At heater outlet is applied an admittance. The damping is considered as the imaginary part of the velocity and the value used is $0.1 \%$ of the real part.

The modes around $53 \mathrm{~Hz}$ are $51 \mathrm{~Hz}, 55 \mathrm{~Hz}$, and $59 \mathrm{~Hz}$, as seen in Fig. 11. This indicates the presence of acoustical resonances around $53 \mathrm{~Hz}$, which can be easily excited.

\subsubsection{Plate Vibrations}

High vibration levels at the lateral duct plates are observed during operation. Severe fatigue problems require permanent welding repairs due to strong excitation levels.

A finite-element model of a detailed and full-length lateral duct plate is developed using shell and beam elements for the

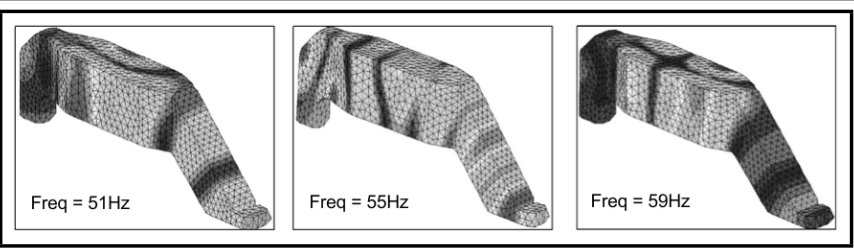

Figure 11. Acoustical modes of the heat exchanger.

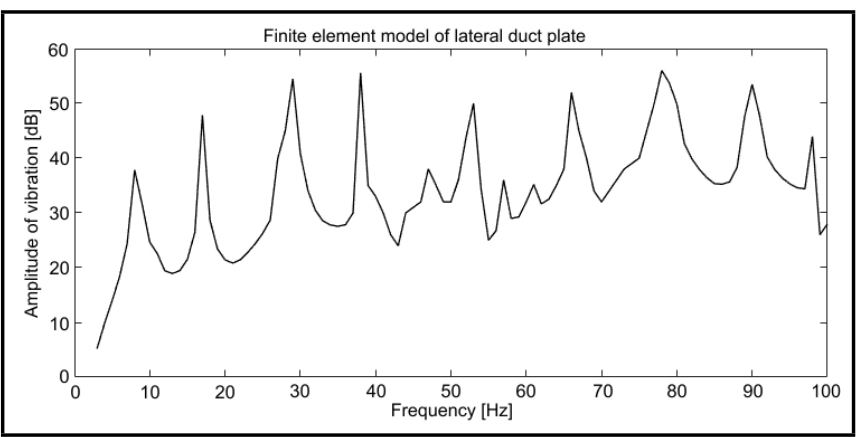

Figure 12. Frequency response function of the lateral duct plate.

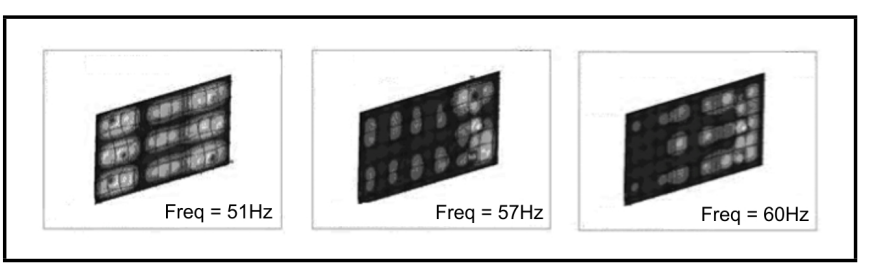

Figure 13. Vibration modes of a lateral duct plate.

reinforcements. A typical frequency response function for this model can be observed in Fig. 12. The presence of a global mode resonance of $51 \mathrm{~Hz}$ can be observed, which is very close to the acoustic mode and the high response frequency $(53 \mathrm{~Hz})$ observed during the measurements.

The three vibration modes with frequencies of around $53 \mathrm{~Hz}$ can be observed in Fig. 13.

\section{RESULTS AND DISCUSSION}

The noise and vibration measurements show a high level of responses at $53 \mathrm{~Hz}$ using a blower fan speed of $550 \mathrm{rpm}$. Through the use of numerical models, an acoustic mode of the heat exchanger, and a mode of plate vibrations have been identified within this frequency band. Beyond this, however, it is important to identify the excitation mechanism.

Eq. (5) is used to calculate the critical speed for fluid-elastic instability to occur, using the data of the heat exchanger under study. The value is found to be $73 \mathrm{~m} / \mathrm{s}$, which is well above the operating speed of the equipment. This shows that fluid-elastic instability is not the excitation mechanism responsible for the flow-induced vibration.

Another possible cause cited in the literature is turbulent buffeting. Eq. (4) is used to obtain values for the frequency at which this phenomenon would occur. Using the data from the heat exchanger under study, these values are found to be above $53 \mathrm{~Hz}$. Thus, turbulent buffeting is not the excitation mechanism for flow-induced vibration.

The last mechanism to be analysed is vortex shedding. Eqs. (1) and (2) are used to calculate the frequency of vortex, which is shedding in this case. Using the flow rate data generated by the fan, the frequency values are found near $53 \mathrm{~Hz}$, indicating that vortex shedding is the likely excitation mechanism for flow-induced vibration. 


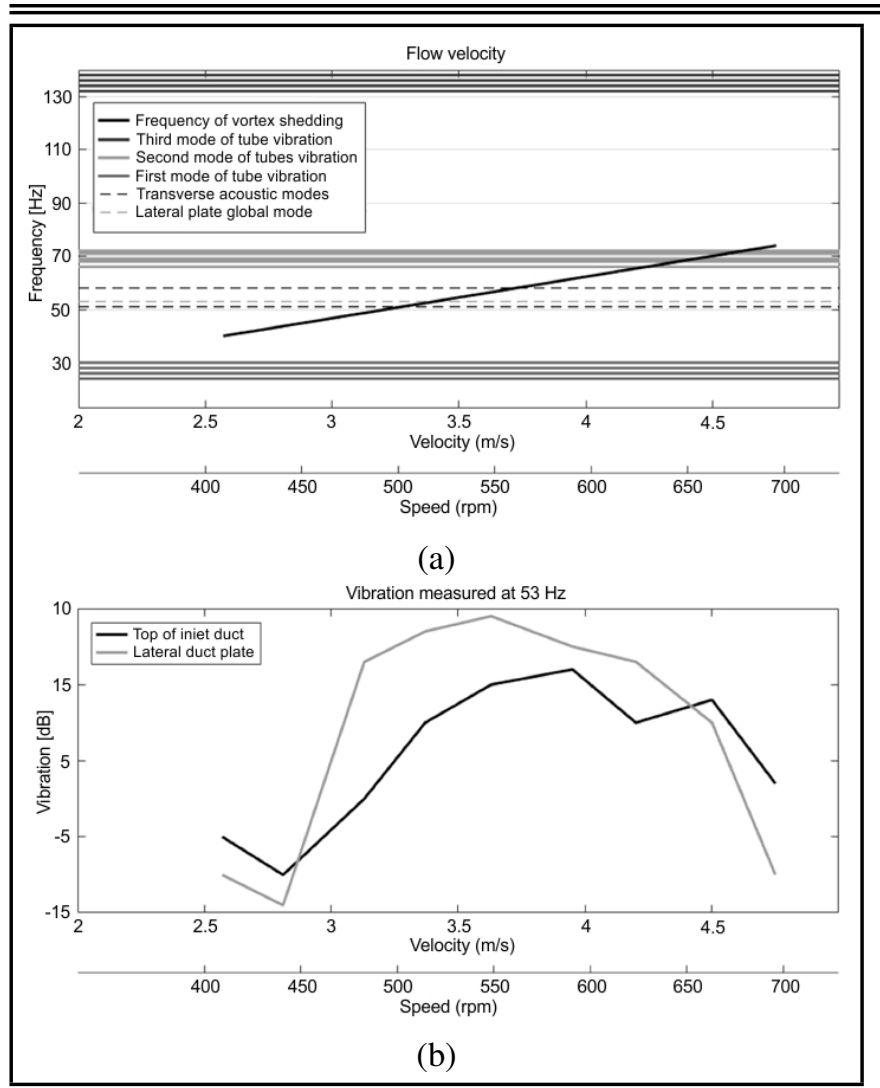

Figure 14. Vortex shedding frequency (a) and the vibration response at $53 \mathrm{~Hz}$, as a function of flow velocity (b)

\subsection{Problem Identification}

Using Eqs. (1) and (2), it is possible to create a graph that correlates the vortex shedding frequency with flow velocity. Figure 14(a) plots the vortex shedding frequency and the natural frequencies of the structure and the acoustical field. The velocity values on the $\mathrm{x}$-axis are indicated by the corresponding blower fan rotation, in revolutions per minute.

At the air velocity of $3.5 \mathrm{~m} / \mathrm{s}$, the vortex shedding frequency coincides with the transverse acoustic mode of the heat exchanger and with a global mode of the side plate, Figure 14(a).

According to Fig. 14(b), it is possible to identify the vibration response measured at lateral duct plate at $53 \mathrm{~Hz}$ for the various fan rotation values. A large response is initiated at an air velocity value of approximately $3.25 \mathrm{~m} / \mathrm{s}$. Around this frequency there is an acoustics mode and the lateral duct plate mode. These modes can be excited by vortex shedding. Tube vibrations do not play a role in this excitation mechanism.

Further evidence of the vortex shedding acoustic mode excitation can be seen from the noise spectra measured at $1 \mathrm{~m}$ from the fan inlet in Fig. 6. After a specific value of fan rotation, the sound pressure level measured at $53 \mathrm{~Hz}$ increases the value. According to Fig. 14(a) there is an acoustic near this frequency. Also, according to Eq. (2) the value of vortex shedding frequency increases with fan rotation.

\subsection{Proposed Solution}

To avoid excitation of the acoustic modes in the heat exchanger, the exchanger width needs to be shortened. For this reason, two longitudinal rows of tubes are removed, and in the empty space, 4-mm-thick plates are placed and welded at the top and bottom borders of the heat exchanger. In Fig. 15, it is possible to identify the location of these plates, also known as acoustic baffles. This procedure does not interfere significantly with the efficiency of the heat exchanger.

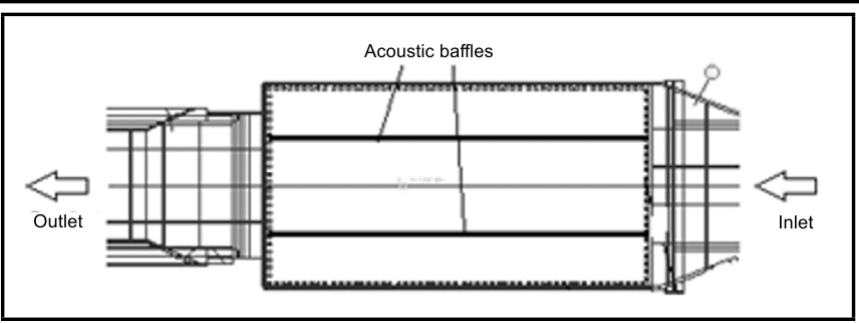

Figure 15. Acoustical baffles in the heat exchanger.

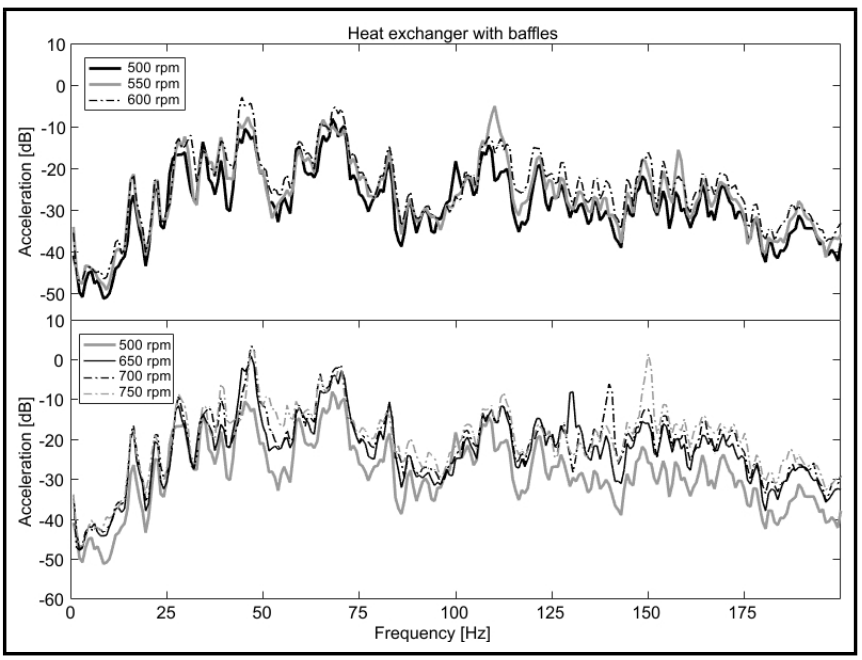

Figure 16. Vibration of lateral plate with acoustical baffles installed inside the heat exchanger.

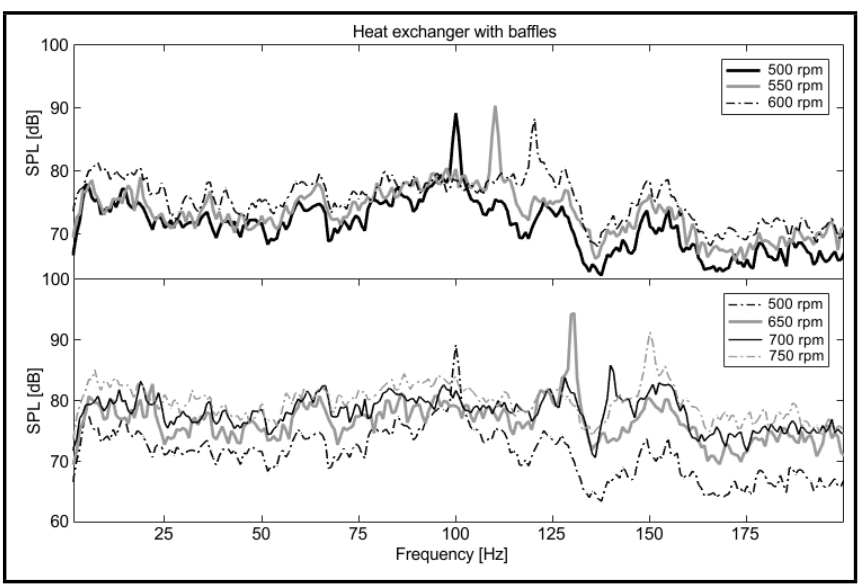

Figure 17. SPL at the inlet of the fan with acoustical baffles installed inside the heat exchanger.

To verify the efficacy of the proposed solution, vibration and noise measurements are taken after the installation of the baffles; the results can be seen in Figs. 16 and 17. The fan rotational speed is varied again from 450 to $750 \mathrm{rpm}$, generating typical air flows inside the heat exchanger. The accelerometer and microphone are placed at the same position as before.

Initially, the vortex shedding mechanism was exciting the acoustic mode at the frequency of $53 \mathrm{~Hz}$. Also, the acoustical resonance excited the lateral duct plate at the same frequency. After installation of the baffle, the acoustical mode is not excited by vortex shedding (see Fig. 17) and the acoustical resonance does not exist to excite the lateral duct plate Fig. 16.

Overall, noise levels at the sugar-refining plant are now considered perfectly acceptable and as expected from this type of industry. The SPL measured at $1 \mathrm{~m}$ from the fan inlet indicates that acoustical resonance disturbances have been completely 
avoided by this modification of the heat exchanger. Some researchers have developed models and methodologies to simulate and optimize this kind of solution. ${ }^{10}$

\section{CONCLUSIONS}

This paper presents some experimental results enabling the identification of a specific frequency of $53 \mathrm{~Hz}$, which is associated with sound and vibration measurements that cause flowinduced vibrations in a heat exchanger. The value of acceleration measured at the lateral plate of the heat exchanger is also high at this frequency, as is the noise measured in front of the fan, which demonstrates the same behaviour. This frequency is correlated with a fan speed of between 500 and $650 \mathrm{rpm}$. This indicates problems caused by flow-induced vibrations at this fan speed.

According to the literature, the main sources of vibration in a heat exchanger are vortex shedding, acoustical resonance, turbulent buffeting and fluid-elastic instability. Using equations from the literature to characterize each source, vortex shedding is identified as the main mechanism for flow-induced vibrations.

Thus, it is necessary to identify which natural frequencies are excited by this mechanism. Models are developed based on the finite-element method. First, a bundle of tubes is modelled to enable the identification of vibration modes and natural frequencies of the heat-exchanger tubes. Following this, the acoustical field inside the heat exchanger is also modelled. Analysis of these results indicates that there are some acoustical modes where the maximum frequency value is close to $53 \mathrm{~Hz}$. A lateral duct plate is also modelled, and through the results, it is possible to identify some vibration modes with natural frequencies close to the frequency of interest.

Using the results of finite-element models and the data on the excitation mechanism, it is possible to assume that vortex shedding excites the acoustic mode of the cavity and the acoustical resonance excites the vibration mode of the plate. The solution to this problem is to insert an acoustic baffle in the cavity and break the acoustic mode. The proposed solution resolves the presented problem, and the subsequent noise and vibration measurements after the installation of the baffles confirm this.

\section{ACKNOWLEDGEMENTS}

The generous financial support of Conselho Nacional de Desenvolvimento Cientco e Tecnolgico (CNPq) and Comisso de Aperfeioamento de Pessoal do Nvel Superior (CAPES) is highly appreciated.

\section{REFERENCES}

1 Cortez, L. A. B. Sugarcane bioethanol - $R \& D$ for productivity and sustainability, Säo Paulo, Blucher. https://doi.org/10.5151/blucheroa-sugarcane

2 Wootton, L. R. Industrial implications of flow induced vibrations, Proc. Institution of Mechanical Engineers, C 416/111, Brighton, England, (1991).

3 Gelbe, H., Jahr, M., and Schrder, K. Flow-induced vibrations in heat exchanger tube bundles, Chem. Eng. Proc., 34, 289-298, (1995). https://doi.org/10.1016/02552701(94)04016-8

4 Padoussis, M. P. A review of flow-induced vibrations in reactor and reactor components, Nucl. Eng. Des., 74, 31-60, (1983). https://doi.org/10.1016/0029-5493(83)90138-3
5 Gorman, D. J. Experimental development of design criteria to limit liquid cross-flow-induced vibration in nuclear reactor heat exchanger equipment, Nucl. Sci. Eng., 61 (3), 324-336, (1976).

6 Weaver, D. S., Lian, H. Y., and Huang, X. Y. Vortex shedding in rotated square tube arrays, J. Fluids Struc., 7, 107121, (1993). https://doi.org/10.1006/jfls.1993.1009

7 Goyder, H. G. D. Acoustic modes in heat exchangers, ASME PVP, 420-2 (107), 114, (2001).

8 Owen, P. R. Buffeting excitation of boiler tube vibration, J. Mech. Eng. Sci., 4-2, 431-439, (1965). https://doi.org/10.1243/jmes_ jour_ 1965_007_065_02

9 Lever, J. H. and Weaver, D. S. A theoretical model for the fluidelastic instability in heat exchanger yube bundles, J. Press. Vess. Technol., 104, 147-158, (1982). https://doi.org/10.1115/1.3264196

10 Eisinger, F. L. and Sullivan, R. E. Suppression of acoustic waves in steam generator and heat exchanger Tube Banks, Journal of Pressure Vessel Technology, 125, 221227, (2003). https://doi.org/10.1115/1.1565079

11 Goyder, H. G. D. Flow-induced vibration in heat exchangers, Trans IChemE, 80 (A), 226-232, (2002). https://doi.org/10.1205/026387602753581971

12 Ziada, S., Oengoerem, A., and Buehlman, E.T. On acoustical resonance in tube arrays Part I: Experiments, J. Fluids Struc., 3, 293-314, (1989). https://doi.org/10.1016/s08899746(89)90083-2

13 Ziada, S., and Oengoerem, A. Vorticity shedding and acoustic resonance in an in-line tube bundle-part I: Vorticity shedding, J. Fluids Struc., 6, 271-292, (1992). https://doi.org/10.1016/0889-9746(92)90010-z

14 Chenoweth, J. M., Chisholm, D., Cowie, R. C., Harris, D., Illingworth, A., Loncaster, J. F., Morris, M., Murray, I., North, C., Ruiz, C., Saunders, E. A. D., Shipes, K.V., Dennis, U., and Webb, R. L. Heat exchanger design handbook $H E D H$. Hemisphere Publishing Corporation, (1993).

15 Pettigrew, M.J., and Taylor, C.E. Vibration analysis of shell-and-tube heat exchangers: An overviewPart 2: vibration response, fretting wear, guidelines, J. Fluids Struc., 18, 485-500, (2003). https://doi.org/10.1016/j.jfluidstructs.2003.08.008

16 Weaver, D. S., and Grover, L. K. Cross flow induced vibrations in a tube bank - turbulent buffeting and fluidelastic instability. J. Sound and Vibration, 59, 277-294, (1978). https://doi.org/10.1016/0022-460x(78)90506-0

17 Owen P. R. Buffeting excitation of boiler tube vibration, J. Mech. Eng. Sci., 7, 431-439, (1965). https://doi.org/10.1243/jmes _ jour_ 1965_007_065_02

18 Price, S. J. An investigation on the Connors equation to predict fluid elastic instability in cylinder arrays. J. Press. Vess. Technol., 123, 448-453, (2001). https://doi.org/10.1115/1.1403445

19 Ziada, S. Vorticity shedding and acoustic resonance in tube bundles, J. Brazilian Society of Mechanical Science and Engineering, 28 (2), 186-199, (2006). https://doi.org/10.1590/s1678-58782006000200008 